\title{
On the fracture of high temperature alloys by creep cavitation under uniaxial or biaxial stress states
}

\author{
John W. Sanders ${ }^{a}$, Mohsen Dadfarnia ${ }^{a, c}$, James Stubbins ${ }^{b, c,{ }^{*}}$, and Petros Sofronis ${ }^{a, c}$ \\ ${ }^{a}$ Department of Mechanical Science and Engineering, University of Illinois at Urbana- \\ Champaign, 1206 West Green Street, Urbana, Illinois 61801 \\ ${ }^{b}$ Department of Nuclear, Plasma, and Radiological Engineering, University of Illinois at \\ Urbana-Champaign, 104 South Wright Street, Urbana, Illinois 61801
}

${ }^{c}$ International Institute for Carbon-Neutral Energy Research (WPI-I2CNER), Kyushu University, 744 Motooka, Nishi-ku, Fukuoka, Fukuoka 819-0395, Japan

\begin{abstract}
It is well known that creep rupture in high temperature alloys is caused by grain boundary cavitation: the nucleation, growth, and coalescence of voids along grain boundaries. However, it has been observed recently that the multiaxial rupture behavior of a promising class of high temperature alloys (Tung et al., 2014) cannot be captured by a well-known empirical creep rupture model due to Hayhurst. In an effort to gain a better understanding of rupture in these materials, we depart from empirical models and simulate the underlying rupture mechanisms directly, employing two related models of void growth from the literature: one due to Sham and Needleman (1983), and an extension of Sham and Needleman's model due to Van der Giessen et al. (1995). Our results suggest that the experimental observations might be explained in terms of the interplay between bulk creep and gain boundary diffusion processes. Furthermore, we find that Sham and Needleman's original void growth model, combined with our rupture model, is well suited to capture the experimental data considered here. Such a mechanism-based understanding of the influence of multiaxial stress states on the creep rupture behavior of high temperature alloys promises to be of value and to provide a basis for the qualification of these alloys for extended service in a variety of elevated temperature applications.
\end{abstract}

*Corresponding author: jstubbin@illinois.edu 


\section{Introduction}

There are a number of elevated temperature applications where the long term durability of metallic alloys limits the design and service lives. This can be due to a number of factors including strength and corrosion concerns. However, a major limitation is the resistance to creep and creep-rupture under anticipated service conditions which may extend to $950^{\circ} \mathrm{C}$ or above. These conditions are of interest for high temperature energy systems and include applications where thermo-chemical processing becomes an attractive alternative to electricity generation. One example is the construction of Generation IV nuclear power plants, where the leading design is the Very High Temperature Reactor (VHTR), so called because its target operating temperatures lie somewhere in the range of $650^{\circ} \mathrm{C}-950^{\circ} \mathrm{C}$. Candidate materials currently under consideration include nickel-based Alloy 230 and Alloy 617. The limiting chemical compositions of Alloys 230 and 617 can be found in [1]. A major limitation to this and other high temperature energy conversion systems is ability of the material to resist creep deformation over very long periods of service, extending to 60 years. In addition, service loading conditions include multiaxial stress states, while standard materials testing is typically performed on uniaxially loaded specimens. Design considerations include strict limitations on creep strain, typically less than $1 \%$, and limitations on time to tertiary creep as well as time to rupture over the entire component service life. Clearly, it is important that we understand the mechanisms by which these materials might fail under service operating conditions. Such an understanding will assist in the certification of these alloys, which will in turn enable engineers to design safe and reliable components for long-term, elevated temperature service.

To date, design of components is based on the conventional approach of consulting a plot of some measure of the applied stress $\sigma$ versus rupture time $t_{r}$ (or other limiting parameters) for a given material at a given temperature, developed from experimental data such as those shown in Figure 1 [1]. When a logarithmic scale is used for both axes, such plots tend to be linear for a given stress state (e.g., uniaxial or biaxial tension) for the stress range considered. Moreover, many times uniaxial and biaxial data are found to be approximately parallel, as they are in Figure 1 (a) for Alloy 230 at $850^{\circ} \mathrm{C}$. This empirical observation led Hayhurst [2] to propose the following relation between stress and rupture time:

$$
A=\left(\alpha \sigma_{I}+3 \beta \sigma_{m}+\gamma \sigma_{e}\right)^{\chi} t_{r}
$$

where $A$ and $\chi$ are material parameters independent of the stress state, $\alpha, \beta$, and $\gamma$ are fitting constants which sum to unity, $\sigma_{I}$ is the maximum principal stress, $\sigma_{m}=\sigma_{k k} / 3$ is the mean stress $^{1}$, and $\sigma_{e}=\sqrt{(3 / 2) \sigma_{i j}^{\prime} \sigma_{i j}^{\prime}}$ is the Mises equivalent stress, with $\sigma_{i j}^{\prime}=\sigma_{i j}-\sigma_{m} \delta_{i j}$ the components of the deviatoric stress and $\delta_{i j}$ the Kronecker delta. Note that the form of (1) guarantees linear and parallel stress versus rupture time behavior when $\sigma_{I}, \sigma_{m}$, and $\sigma_{e}$ are in fixed proportions, as they are for the cases of uniaxial tension and biaxial tension within the wall of an internally pressurized tube.

Recently, Tung et al. [1] observed that their experimental results for internally pressurized tubes made of Alloys 230 and 617 at $850^{\circ} \mathrm{C}$ and $950^{\circ} \mathrm{C}$ were not consistent with (1).

\footnotetext{
${ }^{1}$ Here Einstein summation convention is used, where repeated indices are summed over from 1 to 3.
} 
Of the four alloy/temperature combinations they considered, they observed parallel behavior only for Alloy 230 at $850^{\circ} \mathrm{C}$, as shown in Figure 1 (a). For each of the other combinations, their biaxial data and independently obtained uniaxial data were not parallel, as shown in Figure 1 (b), (c), and (d). Furthermore, nonlinear rupture behavior has also been reported by Abe [3] for 9Cr1Mo steel. Such behavior cannot be captured by (1). Tung et al. speculate in [1] that this behavior might be associated with specimen size effects arising from the formation of voids within the tube walls. It is possible that this size effect is directly related to the stress state itself (e.g., differences in the triaxiality between uniaxial and biaxial tension). In an attempt to gain a better understanding of this behavior, we depart from phenomenological models such as (1) and instead simulate the underlying rupture mechanisms directly.

Rupture in structural metals at high temperatures is known to be caused by creep cavitation (i.e., the nucleation, growth, and coalescence of voids) along grain boundaries [1], [4]. Over the last several decades, a great deal of effort has been put into arriving at a model for grain boundary void growth in creeping solids. The seminal work of Hull and Rimmer [5] observed that void growth occurs under the influence of grain boundary diffusion and surface diffusion. Chuang et al. [6] developed analytical expressions for cavity growth rates assuming surface diffusion and grain boundary diffusion to be the dominant mechanisms. Needleman and Rice [7] were the first to study how bulk creep deformation interacts with grain boundary diffusion to bring about void growth. In particular, they simulated the growth of axisymmetric voids in uniaxial tension under the influence of both grain boundary diffusion and bulk creep numerically, using the finite element method. The seminal contribution of Needleman and Rice was the introduction of a diffusion length $L$, such that the ratio of $L$ to the average cavity radius $a$ is a measure of the relative importance of grain boundary diffusion versus bulk creep. When this ratio is large (typically 20 or greater), grain boundary diffusion is the driving mechanism for void growth, and when this ratio is small (typically 4 or less), bulk creep is the driving mechanism. For intermediate values, the effects of diffusion and bulk creep are comparable, and this interplay between the two mechanisms is at the heart of the present paper. Budiansky et al. [8] subsequently developed analytical expressions for the cavity growth rate under the influence of bulk creep by itself. Sham and Needleman [9] went on to extend the work of Needleman and Rice [7] to multiaxial stress states, and proposed a closed-form expression for the cavity growth rate that approximated their numerical results by stitching together the analytical expressions of Chuang et al. [6] (modified according to an observation by Chen and Argon [10]) and Budiansky et al. [8]. This expression of Sham and Needleman was developed for stress triaxialities $\sigma_{m}^{\infty} / \sigma_{e}^{\infty}>1$, where $\sigma_{m}^{\infty}$ and $\sigma_{e}^{\infty}$ are the mean and Mises equivalent stresses, respectively, corresponding to the remotely applied load. Finally, Van der Giessen et al. [11] extended the results of Sham and Needleman [9] to triaxialities $\sigma_{m}^{\infty} / \sigma_{e}^{\infty}<1$ by stitching on additional expressions due to Tvergaard [12], and to higher porosities (i.e., higher void volume fractions) by incorporating additional expressions of their own.

The remainder of this paper is organized as follows. In Section 2, we review the void growth model of Sham and Needleman [9], and its extension by Van der Giessen et al. [11], in detail. In Section 3, we investigate the rupture behavior predicted by these two models in uniaxial tension and biaxial tension, noting the effects of the dominant cavitation mechanism and the stress triaxiality on rupture time. In Section 4, we apply both models in an effort to reproduce Tung et al.'s [1] experimental stress-rupture time data for Alloys 230 and 617, using constitutive parameters inspired by creep test data from Pataky et al. [13], Maldini et al. [14], and Wright and Wright [15]. We then discuss the effectiveness of the two models at capturing 
the experimental creep rupture data. Finally, in Section 5, we conclude with a summary of the insights we have gained into the creep rupture process, and propose a possible explanation for the results observed by Tung et al. [1].

\section{Failure by void growth and coalescence in creeping solids}

A model approach to void growth and coalescence processes in power-law creeping solids often employed in the literature (see for example [6], [16], [17]) is as follows. The constitutive response of the material in uniaxial tension is characterized by the power law relation:

$$
\dot{\varepsilon}=\dot{\varepsilon}_{0}\left(\sigma / \sigma_{0}\right)^{n}
$$

where $\dot{\varepsilon}$ is the creep strain rate, $\sigma$ is the applied stress, $\dot{\varepsilon}_{0}$ and $\sigma_{0}$ are the reference strain rate and stress, respectively, and $n$ is the creep exponent. For three-dimensional stress states the constitutive description of the material is such that the same relationship holds between the effective strain rate, $\dot{\varepsilon}_{e}=\sqrt{2 \dot{\varepsilon}_{i j} \dot{\varepsilon}_{i j} / 3}$, and the Mises equivalent stress $\sigma_{e}$, where $\dot{\varepsilon}_{i j}$ are the components of the creep strain rate tensor [6]. Voids are modeled as spherical cap-shaped regions of radius $a$ and mean half-spacing $b$, as shown in Figure 2. The volume of such a void is given by

$$
V=\frac{4}{3} \pi a^{3} g(\psi)
$$

where the dihedral angle $\psi$ is the angle the void surface makes with the grain boundary, and

$$
g(\psi)=\frac{1}{\sin \psi}\left(\frac{1}{1+\cos \psi}-\frac{1}{2} \cos \psi\right)
$$

Under the influence of the applied stresses, the voids expand from some initial radius of $a_{0}$ according to the following equation:

$$
\dot{a}=\frac{\dot{V}}{4 \pi a^{2} g(\psi)},
$$

which can be obtained by differentiating (3). It is assumed that surface diffusion occurs rapidly enough to maintain the spherical-cap shape of the voids (i.e., $\psi$ is constant). Coalescence, and thus rupture, is taken to occur when the ratio $a / b$ reaches some critical value $\lambda$. All that remains is to specify the volumetric growth rate $\dot{V}$. 


\subsection{The Sham-Needleman model}

One important contribution of Needleman and Rice's work [7] consisted of tabulated values for the volumetric growth rate of the kind of voids described above (in axisymmetric unit cells) in the presence of bulk creep and grain boundary diffusion, which they generated numerically using the finite element method. However, their results were derived under remotely applied (macroscopic) uniaxial tension. Sham and Needleman [9], taking the same approach as Needleman and Rice [7], tabulated similar numerical data for an axisymmetric stress state with greater triaxiality. They further found that they were able to approximate their results by considering the total volumetric growth rate to be the sum of the growth rate due to grain boundary diffusion and that due to bulk creep deformation:

$$
\dot{V}=\dot{V}^{\mathrm{df}}+\dot{V}^{\mathrm{cr}}
$$

where the grain boundary diffusion term, based on the work of Chuang et al. [6] and Chen and Argon [10], is given by

$$
\frac{\dot{V}^{\mathrm{df}}}{\dot{\varepsilon}_{\infty} a^{3}}=4 \pi\left(\frac{L}{a}\right)^{3}\left[\frac{\sigma_{I}^{\infty} / \sigma_{e}^{\infty}-(1-f) \sigma_{s} / \sigma_{e}^{\infty}}{\ln (1 / f)-(3-f)(1-f) / 2}\right],
$$

and the bulk creep term, based on the work of Budiansky et al. [8], is given by

$$
\frac{\dot{V}^{\mathrm{cr}}}{\dot{\varepsilon}_{\infty} a^{3}}=2 \pi g(\psi)\left[\alpha_{n}\left(\frac{\sigma_{m}^{\infty}}{\sigma_{e}^{\infty}}\right)+\beta_{n}\right]^{n} .
$$

In the above equations, $\sigma_{I}^{\infty}, \sigma_{m}^{\infty}$, and $\sigma_{e}^{\infty}$ are the maximum principal stress, mean stress, and Mises equivalent stress, respectively, corresponding to the remotely applied load, in the axisymmetric cell calculations. For the application of the models, the remote effective strain rate $\dot{\varepsilon}_{\infty}$ is related to $\sigma_{e}^{\infty}$ through (2). The diffusion length $L$ introduced by Needleman and Rice [7] is given by

$$
L=\left(D \sigma_{e}^{\infty} / \dot{\varepsilon}_{\infty}\right)^{1 / 3}
$$

where

$$
D=D_{0} \delta e^{-Q / R T} \Omega / k T
$$

is a quantity related to the grain boundary diffusion coefficient, in which $D_{0} \delta$ is a preexponential factor, $Q$ is the activation energy for grain boundary diffusion, $R=8.314 \mathrm{~J} / \mathrm{mol} \cdot \mathrm{K}$ is the universal gas constant, $T$ is the absolute temperature, $\Omega$ is the atomic volume, and $k=1.38 \times 10^{-23} \mathrm{~J} / \mathrm{K}$ is Boltzmann's constant. The so-called sintering stress $\sigma_{s}$ at the tip of the cavity is given by 


$$
\sigma_{s}=2 \gamma_{s} \sin \psi / a
$$

where $\gamma_{s}$ is the surface energy. The factor $f$ is given by

$$
f=\max \left[\left(\frac{a}{b}\right)^{2},\left(\frac{a}{a+1.5 L}\right)^{2}\right]
$$

and $\alpha_{n}$ and $\beta_{n}$ are given by the following:

$$
\alpha_{n}=3 n / 2, \quad \beta_{n}=(n-1)(n+0.4319) / n^{2} .
$$

It should be noted that (6), (7), and (8) were found to be accurate for $a / L \leq 10, \sigma_{s} / \sigma_{e}^{\infty} \leq 4$, and triaxialities $\sigma_{m}^{\infty} / \sigma_{e}^{\infty}>1$.

\subsection{Van der Giessen et al. 's model}

Van der Giessen et al. [11] also considered spherical cap-shaped voids growing in the presence of both grain boundary diffusion and bulk power-law creep. They extended the model of Sham and Needleman [9] to lower triaxialities $\left(\sigma_{m}^{\infty} / \sigma_{e}^{\infty}<1\right)$ and higher porosities, when void-void interactions become important. To account for lower triaxialities, they incorporated work by Tvergaard [12], who had proposed a linearized form of (8) valid for $\sigma_{m}^{\infty} / \sigma_{e}^{\infty}<1$. To account for higher porosities, they modified Budiansky et al.'s [8] approach, which assumed a single void in an infinite matrix, instead considering each void to be embedded within an infinitely long cylinder of radius $b$. Their final result is as follows. The total growth rate is taken to be the maximum of a "Low-porosity" term (hence the subscript L) and a "High-porosity" term (hence the subscript $\mathrm{H})$ :

$$
\dot{V}=\max \left(\dot{V}_{\mathrm{L}}^{\mathrm{df}}+\dot{V}_{\mathrm{L}}^{\mathrm{cr}}, \dot{V}_{H}^{\mathrm{df}}+\dot{V}_{H}^{\mathrm{cr}}\right)
$$

For low porosities, $\dot{V}_{\mathrm{L}}^{\mathrm{df}}$ is given by (7), and

$$
\frac{\dot{V}_{\mathrm{L}}^{\mathrm{cr}}}{\dot{\varepsilon}_{\infty} a^{3}}=\left\{\begin{array}{cc}
2 \pi g(\psi) m\left[\alpha_{n}\left(\sigma_{m}^{\infty} / \sigma_{e}^{\infty}\right)+\beta_{n}(m)\right]^{n} & \text { if } \sigma_{m}^{\infty} / \sigma_{e}^{\infty} \geq 1 \\
2 \pi g(\psi)\left[\alpha_{n}+\beta_{n}(m)\right]^{n}\left(\sigma_{m}^{\infty} / \sigma_{e}^{\infty}\right) & \text { if } \sigma_{m}^{\infty} / \sigma_{e}^{\infty}<1
\end{array}\right.
$$

where the first term is identical to (8) for mean stresses $\sigma_{m}^{\infty} \geq 0$, and the second term is the linearized form proposed by Tvergaard [12]. For high porosities (corresponding in their analysis

to each void being embedded within an infinitely long cylinder of radius $b$ ), $\dot{V}_{H}^{\text {df }}$ is given by (7) with $f$ simply given by $f=(a / b)^{2}$, rather than by (12), and 


$$
\frac{\dot{V}_{H}^{\mathrm{cr}}}{\dot{\varepsilon}_{\infty} a^{3}}=\left\{\begin{array}{l}
2 \pi g(\psi) m\left[\frac{\alpha_{n}\left(\sigma_{m}^{\infty} / \sigma_{e}^{\infty}\right)+m / n}{1-(0.87 a / b)^{3 / n}}\right]^{n} \quad \text { if } \sigma_{m}^{\infty} / \sigma_{e}^{\infty} \geq 1 \\
2 \pi g(\psi)\left[\frac{\alpha_{n}+m / n}{1-(0.87 a / b)^{3 / n}}\right]^{n}\left(\frac{\sigma_{m}^{\infty}}{\sigma_{e}^{\infty}}\right) \text { if } \sigma_{m}^{\infty} / \sigma_{e}^{\infty}<1
\end{array}\right.
$$

Here $m=\operatorname{sgn}\left(\sigma_{m}^{\infty}\right)$, and

$$
\beta_{n}(m)= \begin{cases}(n-1)(n+0.4319) / n^{2} & \text { if } \sigma_{m}^{\infty} \geq 0 \\ (n-1)(n+0.4031) / n^{2} & \text { if } \sigma_{m}^{\infty}<0\end{cases}
$$

Van der Giessen et al. [11] supposed that the maximum in (14) would switch from low- to highporosity terms when $a / b$ became sufficiently large. In practice, however, we have found that it is possible for the high-porosity term to be larger initially, with the maximum switching to the low-porosity term later on. In some cases, the maximum switched between low- and highporosity terms several times throughout the course of a single simulation. Presumably, such behavior was not intended.

We would like to emphasize here that Van der Giessen et al.'s model [11] is a direct extension of Sham and Needleman's model [9]. For low porosities, high stress triaxialities ( $\sigma_{m}^{\infty} / \sigma_{e}^{\infty}>1$ ), and positive remote mean stresses, the volumetric void growth rate proposed by Van der Giessen et al. [11] is identical to that of Sham and Needleman [9]. The modifications proposed by Van der Giessen et al. [11] come into play for (i) high porosities, (ii) low stress triaxialities $\left(\sigma_{m}^{\infty} / \sigma_{e}^{\infty}<1\right)$, and (iii) negative remote mean stresses (note, however, that negative remote mean stresses will not be considered in the present work). Of particular interest here is whether these modifications have a significant effect on the rupture time predictions for uniaxial tension and biaxial tension within the walls of an internally pressurized tube. We will show shortly that for these two stress states, in which the stress triaxiality is less than one, the volumetric growth rate of Van der Giessen et al. [11] differs from that of Sham and Needleman [9], and this difference does have a marked effect on the predicted rupture times.

\section{Model predictions in uniaxial and biaxial tension}

In this section, we present model predictions for rupture time in uniaxial tension and biaxial tension, the latter case representing the stress state in the wall of an internally pressurized, circular tube. Henceforth, we will neglect any anisotropies associated with the tube stock forming process and assume that the material is isotropic in both uniaxial tension and biaxial tension.

In uniaxial tension, there is only one nonzero stress component: the axial stress $\sigma_{z z}$, as shown in Figure 3 (a). While the applied load $P$ is fixed during a creep rupture test, the axial stress will in general increase during the course of the test due to a decrease in the cross sectional area $A$ of the test specimen caused by incompressible creep deformation. It is straightforward to show (see Appendix A for the details) that the stress is given by 


$$
\sigma_{z z}=\left(P / A_{0}\right) e^{\varepsilon_{e}}
$$

where $P / A_{0}$ is the nominal stress ( $A_{0}$ being the original cross-sectional area), and

$$
\varepsilon_{e}=\int_{0}^{t} \dot{\varepsilon}_{e}(\tau) d \tau
$$

is the total accumulated creep strain. In this case, $\sigma_{I}^{\infty}=\sigma_{e}^{\infty}=\sigma_{z z}$ and $\sigma_{m}^{\infty}=\sigma_{z z} / 3$, from whence it follows that

$$
\sigma_{I}^{\infty} / \sigma_{e}^{\infty}=1, \quad \sigma_{m}^{\infty} / \sigma_{e}^{\infty}=1 / 3
$$

Notice that these ratios are not sensitive to creep-induced strain, and that the stress triaxiality is less than unity.

Within the wall of a capped, circular cylindrical tube of mean radius $r$ and thickness $h$ under internal pressure $p$, there are in general three nonzero stress components: the radial stress $\sigma_{r r}$, the hoop stress $\sigma_{\theta \theta}$, and the axial stress $\sigma_{z z}$. These are illustrated in Figure 3 (b). Under the thin-walled tube approximation, these stress components are given below:

$$
\sigma_{r r}=0, \quad \sigma_{\theta \theta}=2 \sigma_{z z}=p r / h
$$

We pause here to note that the tubes tested by Tung et al. [1] had inner and outer radii of 2.032 $\mathrm{mm}$ and $2.286 \mathrm{~mm}$, respectively. This means that the thickness was roughly $11 \%$ of the outer radius, and so the thin-walled approximation is valid. While the internal pressure $p$ is fixed during typical tube rupture tests, the hoop and axial stresses will in general increase due to an increase in the mean radius $r$ and a decrease in the thickness $h$ caused by creep straining. It is straightforward to show (again, see Appendix A for the details) that the ratio $r / h$ is given by

$$
r / h=\left(r_{0} / h_{0}\right) e^{\sqrt{3} \varepsilon_{e}}
$$

where $r_{0}$ and $h_{0}$ are the original radius and thickness, respectively, and $\varepsilon_{e}$ is the total accumulated creep strain given by (19). The nominal stresses are calculated from (21) with $r=r_{0}$ and $h=h_{0}$. Expressions (21) give rise to the following:

$$
\sigma_{I}^{\infty}=p r / h, \quad \sigma_{m}^{\infty}=p r / 2 h, \quad \sigma_{e}^{\infty}=\sqrt{3} p r / 2 h
$$

Notice that $\sigma_{e}^{\infty}$ is directly proportional to the internal pressure $p$. From (23), the following ratios are obtained:

$$
\sigma_{I}^{\infty} / \sigma_{e}^{\infty}=2 / \sqrt{3} \approx 1.15, \quad \sigma_{m}^{\infty} / \sigma_{e}^{\infty}=1 / \sqrt{3} \approx 0.577
$$


Again, these ratios are not affected by creep. Note how close $\sigma_{I}^{\infty} / \sigma_{e}^{\infty}$ here is to unity, as in (20). In addition, the triaxiality under biaxial tension is larger than that in uniaxial tension by about $73 \%$, though it is still less than unity. The importance of these observations will be discussed shortly.

For a given stress state, we can find the time to rupture $t_{r}$ by integrating (5):

$$
t_{r}=4 \pi g(\psi) \int_{a_{0}}^{\lambda b} \frac{a^{2}}{\dot{V}(a, \sigma)} d a
$$

where $\dot{V}$ is the volumetric growth rate of either Sham and Needleman (6) or Van der Giessen et al. (14). Again, these two models give different results for the same remote stress state when the porosity is large, and when the stress triaxiality is low. In general, the integration in (25) must be carried out numerically. Henceforth, for all simulations, the reference strain rate is taken to be $\dot{\varepsilon}_{0}=1 \times 10^{-6} \mathrm{~s}^{-1}$ (note that this choice sets the numerical values of $\sigma_{0}$ reported in this work), and the various material parameters are assigned values representative of nickel-chrome alloys shown in Table 1 (note that we have assumed an initial void radius of $a_{0}=0.5 \mu \mathrm{m}$, and we have chosen the following values: $b=50 \mu \mathrm{m}, \lambda=0.5$ ). In the case of the biaxial stress state (i.e., the internally pressurized tube), the mean radius and thickness are taken to be those of Tung et al. [1]: $r=2.159 \mathrm{~mm}$ and $h=0.254 \mathrm{~mm}$, respectively.

Figure 4 shows representative void radius histories toward fracture for nominal remote equivalent stress $\sigma_{e}^{\infty}=35 \mathrm{MPa}, \sigma_{0}=153 \mathrm{MPa}, n=6.75$, and $T=800{ }^{\circ} \mathrm{C}$. It can be seen that for each curve the void radius starts at its assumed initial value of $a_{0}=0.5 \mu \mathrm{m}$, and rupture occurs when $a=b / 2=25 \mu \mathrm{m}$. Furthermore, the curves corresponding to Van der Giessen et al.'s model exhibit abrupt failure after about 1 hour, whereas Sham and Needleman's model results in more stable behavior, with rupture occurring after about 10-100 hours. This difference is to be expected, given that the stress triaxiality for both stress states is less than unity, and therefore the volumetric growth rate of Van der Giessen et al. [11] differs from that of Sham and Needleman [9]. For these simulations, the nominal value of $L / a$ at fracture came out to about 6.3, indicating predominantly bulk creep driven void growth.

We proceed with a parametric study of the model predictions for rupture time by varying the reference stress $\sigma_{0}$. We emphasize that $\sigma_{0}$ does not by itself determine the dominant void growth mechanism (again, the dominant mechanism is determined by the non-dimensionalized diffusion length $L / a$ ): rather, $\sigma_{0}$ is simply one of several parameters that can be varied independently of the others to illustrate the behavior of the two models. Figure 5 shows representative nominal remote equivalent stress versus rupture time results for $n=6.5, T=800$ ${ }^{\circ} \mathrm{C}$, and various values of $\sigma_{0} \in[100 \mathrm{MPa}, 5.5 \mathrm{GPa}]$ using the models of Sham and Needleman [9] and Van der Giessen et al. [11] (we will see in Section 4 that these are realistic parameter values for nickel-chrome alloys). Similar plots to Figure 5, not shown, can be found by varying $n$ or any of the other parameters. Nominal values of $L / a$ at fracture are shown alongside the curves at various points.

From Figure 5, it can be seen that both the Sham-Needleman model [9] and Van der Giessen et al. model [11] exhibit three distinct types of behavior. For smaller values of $L / a$, the curves for uniaxial tension (solid line) and biaxial tension (dashed line) are roughly linear 
and parallel, as they are for the curves labeled $\sigma_{0}=100 \mathrm{MPa}$ in Figure 5 (a) and $\sigma_{0}=200 \mathrm{MPa}$ in Figure 5 (b). This corresponds to void growth in which bulk creep within the grains is the driving mechanism. In the case of Sham and Needleman's model, if we neglect the grain boundary diffusion contribution in (6), and we also neglect the effects of incompressible creep deformation on the stress (as justified in Appendix A), we can perform the integration in (25) analytically and obtain

$$
t_{r}=\frac{2 \sigma_{0}^{n}}{\dot{\varepsilon}_{0}} \ln \left(\frac{\lambda b}{a_{0}}\right)\left[\alpha_{n}\left(\frac{\sigma_{m}^{\infty}}{\sigma_{e}^{\infty}}\right)+\beta_{n}\right]^{-n}\left(\sigma_{e}^{\infty}\right)^{-n}
$$

This confirms that, when grain boundary diffusion is negligible, a plot of $\log \sigma_{e}^{\infty}$ versus $\log t_{r}$ should be linear, with the same slope $(-1 / n)$ for both uniaxial tension and biaxial tension, but with different intercepts arising from the different values of $\sigma_{m}^{\infty} / \sigma_{e}^{\infty}$. This offset between the intercepts of the creep dominated curves appears to be larger for Sham and Needleman's model than it is for Van der Giessen et al.'s model, at least for the triaxialities considered here.

For larger values of $L / a$, the curves for uniaxial tension and biaxial tension are parallel and very nearly collinear, as they are for the curves labeled $\sigma_{0}=2 \mathrm{GPa}$ in Figure 5 (a) and $\sigma_{0}=5.5 \mathrm{GPa}$ in Figure 5 (b). This corresponds to void growth in which grain boundary diffusion is the driving mechanism. In the case of Sham and Needleman's model, if we neglect the bulk creep term in (6) and simplify, we find that (5) reduces to

$$
\dot{a}=\frac{D}{a^{2} g(\psi)}\left[\frac{\sigma_{I}^{\infty}-(1-f) \sigma_{s}}{\ln (1 / f)-(3-f)(1-f) / 2}\right] .
$$

That is, $\dot{a}$ only depends on $\sigma_{I}^{\infty}$ (not on the ratio $\sigma_{I}^{\infty} / \sigma_{e}^{\infty}$ ). This means that, when bulk creep is negligible, a plot of $\log \sigma_{I}^{\infty}$ versus $\log t_{r}$ should be the same for both stress states. A plot of $\log \sigma_{e}^{\infty}$ versus $\log t_{r}$ will be different, but only slightly, due to the fact that the values of $\sigma_{I}^{\infty} / \sigma_{e}^{\infty}$ are so close for the two stress states, as mentioned earlier. This explains the very small offset between the diffusion dominated curves in Figure 5 (a). Note that, when grain boundary diffusion is the driving mechanism for void growth, the lifetime of the material is significantly increased compared to when bulk creep is the driving mechanism. This effect, which can be seen in Figure 5, was first explained by Needleman and Rice [7].

Finally, for intermediate values of $L / a$, the curves for uniaxial tension and biaxial tension are nonlinear, as demonstrated by the curves labeled $\sigma_{0}=250 \mathrm{MPa}$ in Figure 5 (a) and $\sigma_{0}=1 \mathrm{GPa}$ in Figure $5(\mathrm{~b})$. This corresponds to void growth in which the effects of grain boundary diffusion and bulk creep are comparable. In such cases, the behavior transitions from approximately bulk creep dominated cavitation at high stresses to approximately grain boundary diffusion dominated cavitation at low stresses, resulting in a change in the slope of the curves. We note that this is precisely the kind of behavior observed by Tung et al. [1] in their experiments ( $c f$. Figure 1), as well as Abe [3]. We will return to this observation in Section 5. 


\section{Application to Alloys 230 and 617}

We now attempt to reproduce the experimental creep rupture data in Figure 1 using the models we have presented. To do so, it was necessary to choose numerical values for the creep parameters $\sigma_{0}$ and $n$. Normally these parameters can be estimated from uniaxial creep test data. Unfortunately, such data were not available at the same temperatures as the creep rupture data shown in Figure 1 , namely, $850^{\circ} \mathrm{C}$ and $950^{\circ} \mathrm{C}$. We therefore decided to choose the values of $\sigma_{0}$ and $n$ by regression. To get an idea of what reasonable values might be, we consulted experimental creep test data from Pataky et al. [13] and Maldini et al. [14] for Alloy 230 at $800^{\circ} \mathrm{C}$ and $900^{\circ} \mathrm{C}$, and data from Wright and Wright [15] for Alloy 617 at $800^{\circ} \mathrm{C}, 900^{\circ} \mathrm{C}$, and $1000^{\circ} \mathrm{C}$. The relevant experimental parameter values for temperatures $800^{\circ} \mathrm{C}, 900^{\circ} \mathrm{C}$, and $1000^{\circ} \mathrm{C}$ are summarized in Table 2 and Table 3. The values of $\sigma_{0}$ and $n$ used in our simulations for $850^{\circ} \mathrm{C}$ and $950^{\circ} \mathrm{C}$ were found numerically by least-squares regression: in each case, values of $\sigma_{0}$ and $n$ were chosen so as to minimize the sum of the squared error in the time to rupture (for more details on this fitting process, see Appendix B). These optimal values are listed in Table 4 and Table 5, and the nominal stress versus rupture time results obtained using these values are shown in Figure 6. Notice that the optimal parameter values are almost identical for both the Sham and Needleman model [9] and the Van der Giessen et al. model [11].

From Figure 6, it appears that Sham and Needleman's model is better suited to capture the experimental data than that of Van der Giessen et al. [11], owing to the relatively large offset between the curves for uniaxial tension and biaxial stress state mentioned in Section 3. This is surprising, given that the modifications of Van der Giessen et al. [11] apply precisely when the stress triaxiality is less than unity, which is the case for both uniaxial tension and biaxial tension. In other words, we might have expected the model of Van der Giessen et al. [11] to do better than that of Sham and Needleman [9]. We wish to emphasize that this is not necessarily due to any inaccuracies on the part of Van der Giessen et al.'s [11] void growth model. Rather, it is quite possible that the discrepancies between the present model predictions and the experimental data could be attributed to the nature of the rupture model we have used in the present work. Future research might include the use of a more sophisticated rupture model, perhaps accounting for void nucleation effects, which were not considered here. At present, however, on the basis of our results, we would recommend using Sham and Needleman's [9] void growth model to predict rupture times within the framework of the rupture model presented here.

\section{Conclusion}

Creep rupture in high temperature alloys is known to be caused by grain boundary cavitation processes, in which bulk creep and grain boundary diffusion each plays a role. The dominant mechanism in a given circumstance is indicated by the ratio of Needleman and Rice's [7] diffusion length $L$ to the void radius $a$ : for small values, on the order of 4 or less, bulk creep is the driving mechanism; for large values, on the order of 20 or more, grain boundary diffusion is the driving mechanism; and for intermediate values, the effects of both mechanisms are comparable. To date, design of high temperature alloy components is based on the conventional approach of consulting a log-log plot of stress versus rupture time. While such plots tend to be linear for a given material at a given temperature, Tung et al. [1] observed non-parallel behavior 
for different stress states for Alloy 230 at $950^{\circ} \mathrm{C}$, as shown in Figure 1 (b), and for Alloy 617 at $850^{\circ} \mathrm{C}$ and $950^{\circ} \mathrm{C}$, as shown in Figure 1 (c) and (d). Similar nonlinear behavior has also been reported by Abe [3] for $9 \mathrm{Cr}-1 \mathrm{Mo}$ steel. The fracture model presented in this work on the basis of the void growth models of Sham and Needleman [9] and Van der Giessen et al. [11] suggests that the response curves are linear and parallel only when the fracture process is creep or grain boundary diffusion dominated. When the effects of both bulk creep and grain boundary diffusion are comparable, both models predict nonlinear stress versus rupture time behavior. In particular, there is a transition from creep-dominated cavitation at high stresses to grain boundary diffusion-dominated cavitation at low stresses, resulting in a change in the slope of the stress versus rupture time curves, as seen in Figure 5. This suggests that the experimental rupture data obtained by Tung et al. [1] and Abe [3], which could not be captured by a well-known empirical relation due to Hayhurst [2], might be explained in terms of the interplay between bulk creep and grain boundary diffusion. Furthermore, attempts to fit Tung et al.'s [1] data, shown in Figure 6, appear to indicate that Sham and Needleman's model [9] is well suited to capture experimental creep rupture data within the framework of the rupture model presented here. Possible future work might include the use of a more sophisticated creep constitutive relation, taking into account both transient and steady-state creep (e.g., that of Robinson et al. [18]), or a more sophisticated rupture model that accounts for void nucleation effects.

\section{Acknowledgements}

The authors would like to express their gratitude to Hsiao-Ming Tung, Kun Mo, Garrett Pataky, Huseyin Sehitoglu, and Maurizio Maldini for sharing invaluable experimental data, without which this work would not have been possible. This work was partially supported by DOENEUP under the Integrated Research Project (IRP) entitled, "Fuel Aging in Storage and Transportation (FAST)."

\section{References}

[1] H.-M. Tung, K. Mo and J. F. Stubbins, "Biaxial thermal creep of Inconel 617 and Haynes 230 at 850 and 950C," J. Nucl. Mater., vol. 447, pp. 28-37, 2014.

[2] D. R. Hayhurst, "Creep rupture under multi-axial states of stress," J. Mech. Phys. Solids, vol. 20, pp. 381-390, 1972.

[3] F. Abe, "Creep behavior, deformation mechanisms, and creep life of Mod.9Cr-1Mo steel," Metallurgical and Materials Transactions A, vol. 46A, pp. 5610-5625, 2015.

[4] A. S. Argon, "Mechanisms and mechanics of fracture in creeping alloys," Recent Advances in Creep and Fracture of Engineering Materials and Structures, pp. 1-52, 1982.

[5] D. Hull and D. E. Rimmer, "The growth of grain-boundary voids under stress," Phil. Mag., vol. 4, pp. 673-687, 1959.

[6] T.-J. Chuang, K. I. Kagawa, J. R. Rice and L. B. Sillis, "Non-equilibrium models for diffusive cavitation of grain interfaces," Acta Metall., vol. 27, pp. 265-284, 1979. 
[7] A. Needleman and J. R. Rice, "Plastic creep flow effects in the diffusive cavitation of grain boundaries," Acta metall., vol. 28, pp. 1315-1332, 1980.

[8] B. Budiansky, J. W. Hutchinson and S. Slutsky, "Void growth and collapse in viscous solids," Mechanics of Solids: The Rodney Hill 60th Anniversary Volume, pp. 607-652, 1982.

[9] T. L. Sham and A. Needleman, "Effects of triaxial stressing on creep cavitation of grain boundaries," Acta metall., vol. 31, no. 6, pp. 919-926, 1983.

[10] I.-W. Chen and A. S. Argon, "Diffusive growth of grain-boundary cavities," Acta Metall., vol. 29, pp. 1759-1768, 1981.

[11] E. Van der Giessen, M. W. D. Van der Burg, A. Needleman and V. Tvergaard, "Void growth due to creep and grain boundary diffusion at high triaxialities," J. Mech. Phys. Solids, vol. 43, no. 1, pp. 123-165, 1995.

[12] V. Tvergaard, "On the creep constrained diffusive cavitation of grain boundary facets," $J$. Mech. Phys. Solids, vol. 32, pp. 373-393, 1984.

[13] G. J. Pataky, H. Sehitoglu and H. J. Maier, "Creep deformation and mechanisms in Haynes 230 at 800C and 900C," J. Nucl. Mater., vol. 443, pp. 484-490, 2013.

[14] M. Maldini, G. Angella and V. Lupinc, "Analysis of Creep Curves of Haynes 230 Superalloy," Mater. Sci. Forum, Vols. 638-642, pp. 2285-2290, 2010.

[15] J. K. Wright and R. N. Wright, "Creep rupture of pressurized alloy 617 tubes INL/EXT-1330251," Idaho National Laboratory, Idaho Falls, Idaho, 2013.

[16] A. C. F. Cocks and M. F. Ashby, "On creep fracture by void growth," Prog. Mater. Sci., vol. 27, pp. 199-244, 1982.

[17] D. R. Hayhurst, J. Lin and R. J. Hayhurst, "Failure in notched tension bars due to hightemperature creep: Interaction between nucleation controlled cavity growth and continuum cavity growth," Int. J. Solids Struct., vol. 45, pp. 2233-2250, 2008.

[18] D. N. Robinson, C. E. Pugh and J. M. Corum, "Constitutive equations for describing hightemperature inelastic behavior of structural alloys," in Proceedings of Specialist Meeting on High-Temperature Structural Design Technology of LMFBRs, IAEA Report IWGFR/11, 1976.

[19] H. J. Frost and M. F. Ashby, Deformation-Mechanism Maps: The Plasticity and Creep of Metals and Ceramics, Pergamon Press, 1982.

[20] R. Chattopadhyay, Surface Wear: Analysis, Treatment, and Prevention, ASM International, 2001.

[21] F. Schubert, U. Bruch, R. Cook, H. Diehl, P. J. Ennis, W. Jakobeit, H. J. Penkalla, E. T. Heesen and G. Ullrich, "Creep rupture behavior of candidate materials for nuclear process heat applications," Nucl. Technol., vol. 66, no. 2, pp. 227-240, 1984. 


\section{Appendix A: Effect of creep deformation on stress}

In this appendix, we quantify the effects of creep straining on the stresses within both a tensile test specimen and an internally pressurized, thin-walled tube.

\section{Uniaxial tension}

In uniaxial tension, the only nonzero stress component is

$$
\sigma_{z z}=P / A
$$

where $P$ is the applied load and $A$ is the instantaneous cross-sectional area. The stress deviator therefore has cylindrical polar component

$$
\sigma_{\theta \theta}^{\prime}=-\sigma_{z z} / 3
$$

It follows that

$$
\dot{\varepsilon}_{\theta \theta}=-\dot{\varepsilon}_{e} / 2,
$$

where $\dot{\varepsilon}_{e}$ is the equivalent strain rate. But

$$
\dot{\varepsilon}_{\theta \theta}=\dot{r} / r
$$

where $r$ is the radius of the specimen (assuming a circular cross section). We thus have that

$$
\dot{r}=-\left(\dot{\varepsilon}_{e} / 2\right) r
$$

from which it follows that

$$
r(t)=r_{0} e^{-\varepsilon_{e}(t) / 2}
$$

where $r_{0}$ is the nominal radius, and

$$
\varepsilon_{e}(t)=\int_{0}^{t} \dot{\varepsilon}_{e}(\tau) d \tau
$$

is the accumulated creep strain. Thus, the instantaneous cross-sectional area is given by

$$
A=\pi r^{2}=A_{0} e^{-\varepsilon_{e}}
$$

where $A_{0}=\pi r_{0}^{2}$ is the nominal area. It follows that the axial stress is given by 


$$
\sigma_{z z}=P / A=\left(P / A_{0}\right) e^{\varepsilon_{e}} .
$$

It can be seen from (A.9) that, as creep strain accumulates, the stress increases exponentially from its nominal value. This is a well-known result.

\section{Biaxial tension (tube)}

Consider a capped, circular cylindrical, thin-walled tube of mean radius $r$ and thickness $h$ under internal pressure $p$. Under the thin-walled approximation, the boundary value problem is statically determinate, and the nonzero cylindrical polar stress components are given by

$$
\sigma_{r r}=0, \quad \sigma_{\theta \theta}=2 \sigma_{z z}=p r / h .
$$

Note that the stresses are proportional to the ratio $r / h$. From (A.10), the stress deviator is found to have cylindrical polar component

$$
\sigma_{\theta \theta}^{\prime}=p r / 2 h,
$$

and the Mises equivalent stress is given by

$$
\sigma_{e}=\sqrt{3} p r / 2 h
$$

It follows that

$$
\dot{\varepsilon}_{\theta \theta}=(\sqrt{3} / 2) \dot{\varepsilon}_{e},
$$

where $\dot{\varepsilon}_{e}$ is the equivalent strain rate. But

$$
\dot{\varepsilon}_{\theta \theta}=\dot{r} / r=-\dot{h} / h .
$$

We therefore have that

$$
\begin{gathered}
\dot{r}=(\sqrt{3} / 2) \dot{\varepsilon}_{e} r \\
\dot{h}=-(\sqrt{3} / 2) \dot{\varepsilon}_{e} h
\end{gathered}
$$

from which it follows that 


$$
\begin{gathered}
r(t)=r_{0} e^{(\sqrt{3} / 2) \varepsilon_{e}(t)} \\
h(t)=h_{0} e^{-(\sqrt{3} / 2) \varepsilon_{e}(t)}
\end{gathered}
$$

where $r_{0}$ and $h_{0}$ are the nominal mean radius and thickness, respectively, and $\varepsilon_{e}(t)$ is the accumulated creep strain as defined previously by (A.7). From (A.16) we find that

$$
r / h=\left(r_{0} / h_{0}\right) e^{\sqrt{3} \varepsilon_{e}}
$$

\section{Estimates for the total accumulated creep strain}

For the application of the rupture model, we identify $\sigma_{e}$ with $\sigma_{e}^{\infty}$ and $\dot{\varepsilon}_{e}$ with $\dot{\varepsilon}_{\infty}$. Thus, in both uniaxial tension and biaxial tension, we may write

$$
\sigma_{e}^{\infty}=\sigma_{e}^{\mathrm{nom}} e^{\xi \varepsilon_{\infty}}
$$

where

$$
\sigma_{e}^{\text {nom }}=\left\{\begin{array}{cc}
P / A_{0} & \text { uniaxial tension } \\
\sqrt{3} p r_{0} / 2 h_{0} & \text { biaxial tension }
\end{array}\right.
$$

is the nominal equivalent stress,

$$
\xi= \begin{cases}1 & \text { uniaxial tension } \\ \sqrt{3} & \text { biaxial tension }\end{cases}
$$

is a constant of order unity, and

$$
\varepsilon_{\infty}=\int_{0}^{t} \dot{\varepsilon}_{\infty}(\tau) d \tau
$$

Now $\dot{\varepsilon}_{\infty}$ is related to $\sigma_{e}^{\infty}$ as follows:

$$
\dot{\varepsilon}_{\infty}=\dot{\varepsilon}_{0}\left(\sigma_{e}^{\infty} / \sigma_{0}\right)^{n}
$$

We therefore have that

$$
\dot{\varepsilon}_{\infty}=\dot{\varepsilon}_{0}\left(\sigma_{e}^{\text {nom }} / \sigma_{0}\right)^{n} e^{n \xi \varepsilon_{\infty}} .
$$


This is a first-order, ordinary differential equation in $\varepsilon_{\infty}$, and it is straightforward to show that, with $\varepsilon_{\infty}(0)=0, \varepsilon_{\infty}(t)$ is given by

$$
\varepsilon_{\infty}(t)=-(1 / n \xi) \ln \left[1-n \xi\left(\dot{\varepsilon}_{0} t\right)\left(\sigma_{e}^{\mathrm{nom}} / \sigma_{0}\right)^{n}\right]
$$

Notice that, for $\dot{\varepsilon}_{0} t_{r} \square 1$ or $\sigma_{e}^{\text {nom }} / \sigma_{0} \square 1$, we may Taylor expand (A.24) to find that

$$
\varepsilon_{\infty}(t)=\left(\dot{\varepsilon}_{0} t\right)\left(\sigma_{e}^{\mathrm{nom}} / \sigma_{0}\right)^{n}+\mathrm{O}\left[\left(\dot{\varepsilon}_{0} t\right)^{2}\left(\sigma_{e}^{\mathrm{nom}} / \sigma_{0}\right)^{2 n}\right]
$$

which in turn implies that

$$
e^{\xi \varepsilon_{\infty}}=1+\mathrm{O}\left[\left(\dot{\varepsilon}_{0} t\right)\left(\sigma_{e}^{\mathrm{nom}} / \sigma_{0}\right)^{n}\right]
$$

Hence, when $\dot{\varepsilon}_{0} t_{r} \square 1$ or $\sigma_{e}^{\text {nom }} / \sigma_{0} \square 1$, the factor $e^{\xi \varepsilon_{\infty}}$ will not vary much from unity, and we may neglect the effects of creep straining on the stresses. We would like to point out that these conditions are met for many of the simulations presented in this work. However, in order to be completely precise, we have included the effects of creep straining in all of our calculations.

\section{Appendix B: Numerical fitting procedure}

In order to fit each rupture model to experimental data, as shown in Figure 6, the following procedure was employed. For a given alloy, temperature, and void growth model, the sum of the squared errors in rupture time

$$
S\left(\sigma_{0}, n\right)=\sum_{i}\left[t_{r, i}^{\mathrm{pred}}\left(\sigma_{0}, n\right)-t_{r, i}^{\mathrm{exp}}\right]^{2}
$$

where $t_{r, i}^{\text {pred }}$ is the numerically predicted rupture time, $t_{r, i}^{\exp }$ is the experimentally observed rupture time, and the sum ranges over both uniaxial and biaxial data points, is regarded as a function of the alloy- and temperature-dependent parameters $\sigma_{0}$ and $n$. When $S$ is plotted against $\sigma_{0}$ and $n$, the result is a two-dimensional surface in parameter space. These parameters are chosen so as to minimize $S$ within certain domains. The domains used for Alloy 230 were $\sigma_{0} \in[55 \mathrm{MPa}, 155 \mathrm{MPa}]$ for $850^{\circ} \mathrm{C}, \sigma_{0} \in[15 \mathrm{MPa}, 75 \mathrm{MPa}]$ for $950^{\circ} \mathrm{C}$, and $n \in[3.4,7.7]$ for both temperatures (these values were inspired by data from Pataky et al. [13] and Maldini et al. [14]; see Table 2). The domains used for Alloy 617 were $\sigma_{0} \in[80 \mathrm{MPa}, 130 \mathrm{MPa}]$ for $850^{\circ} \mathrm{C}$, $\sigma_{0} \in[30 \mathrm{MPa}, 80 \mathrm{MPa}]$ for $950^{\circ} \mathrm{C}$, and $n \in[5,7]$ for both temperatures (these values were inspired by data from Wright and Wright [15]; see Table 3). In the present case, since there is no closed-form expression for $t_{r, i}^{\text {pred }}$, the minimization was done numerically by direct inspection of the surface defined by $S$. The optimal values can be found in Table 4 and Table 5. 


\section{Tables and Figures}

Table 1. Parameter and material values used in all simulations. The material values are representative of Ni-Cr alloys. Here we have assumed an initial void radius of $a_{0}=0.5 \mu \mathrm{m}$, and we have chosen the following values: $b=50 \mu \mathrm{m}, \lambda=a / b=0.5$.

\begin{tabular}{ccc}
\hline Parameter & Symbol & Value \\
\hline Initial void radius & $a_{0}$ & $0.5 \mu \mathrm{m}$ \\
Void half-spacing & $b$ & $50 \mu \mathrm{m}$ \\
Critical $a / b$ ratio & $\lambda$ & 0.5 \\
Dihedral angle & $\psi$ & $70^{\circ}[7]$ \\
Pre-exponential factor & $D_{0} \delta$ & $2.8 \times 10^{-15} \mathrm{~m}^{3} / \mathrm{s}[19]$ \\
Activation energy & $Q$ & $115 \mathrm{~kJ} / \mathrm{mol}^{2}[19]$ \\
Atomic volume & $\Omega$ & $1.1 \times 10^{-29} \mathrm{~m}^{3}[19]$ \\
Surface energy & $\gamma_{s}$ & $0.5 \mathrm{~J} / \mathrm{m}^{2}[20]$ \\
\hline
\end{tabular}

Table 2. Material parameter values for Alloy 230 at $800^{\circ} \mathrm{C}$ and $900^{\circ} \mathrm{C}$, based on experimental data from Pataky et al. [13] and Maldini et al. [14]. The reference strain rate is $\dot{\varepsilon}_{0}=1 \times 10^{-6} \mathrm{~s}^{-1}$.

\begin{tabular}{cccccc}
\hline & \multicolumn{3}{c}{ Maldini et al. [14] } & \multicolumn{2}{c}{ Pataky et al. [13] } \\
Alloy & Temperature & $\sigma_{0}[\mathrm{MPa}]$ & $n$ & $\sigma_{0}[\mathrm{MPa}]$ & $n$ \\
\hline \multirow{2}{*}{230} & $800^{\circ} \mathrm{C}$ & 153 & 6.75 & 92.1 & 5.19 \\
& $900^{\circ} \mathrm{C}$ & 75.9 & 7.73 & 6.32 & 3.40 \\
\hline
\end{tabular}

Table 3. Material parameter values for Alloy 617 at $800^{\circ} \mathrm{C}, 900^{\circ} \mathrm{C}$, and $1000^{\circ} \mathrm{C}$, based on experimental data from Wright and Wright [15]. The reference strain rate is $\dot{\varepsilon}_{0}=1 \times 10^{-6} \mathrm{~s}^{-1}$.

\begin{tabular}{cccc}
\hline Alloy & Temperature & $\sigma_{0}[\mathrm{MPa}]$ & $n$ \\
\hline \multirow{6}{*}{617} & $800^{\circ} \mathrm{C}$ & 122 & 6.94 \\
& $900^{\circ} \mathrm{C}$ & 62.5 & 5.94 \\
& $1000^{\circ} \mathrm{C}$ & 35.1 & 5.17 \\
\hline
\end{tabular}


Table 4. Optimal material parameter values for Sham and Needleman's model (chosen by regression), used for the simulations in Figure 6. The reference strain rate is $\dot{\varepsilon}_{0}=1 \times 10^{-6} \mathrm{~s}^{-1}$.

\begin{tabular}{cccc}
\hline Alloy & Temperature & $\sigma_{0}[\mathrm{MPa}]$ & $n$ \\
\hline \multirow{2}{*}{230} & $850^{\circ} \mathrm{C}$ & 155 & 3.40 \\
& $950^{\circ} \mathrm{C}$ & 75.0 & 4.91 \\
\hline \multirow{2}{*}{617} & $850^{\circ} \mathrm{C}$ & 130 & 5.00 \\
& $950^{\circ} \mathrm{C}$ & 80.0 & 5.00 \\
\hline
\end{tabular}

Table 5. Optimal material parameter values for Van der Giessen et al.'s model (chosen by regression), used for the simulations in Figure 6. The reference strain rate is $\dot{\varepsilon}_{0}=1 \times 10^{-6} \mathrm{~s}^{-1}$.

\begin{tabular}{cccc}
\hline Alloy & Temperature & $\sigma_{0}[\mathrm{MPa}]$ & $n$ \\
\hline \multirow{2}{*}{230} & $850^{\circ} \mathrm{C}$ & 155 & 3.40 \\
& $950^{\circ} \mathrm{C}$ & 75.0 & 3.40 \\
\hline \multirow{2}{*}{617} & $850^{\circ} \mathrm{C}$ & 130 & 5.00 \\
& $950^{\circ} \mathrm{C}$ & 80.0 & 5.00 \\
\hline
\end{tabular}




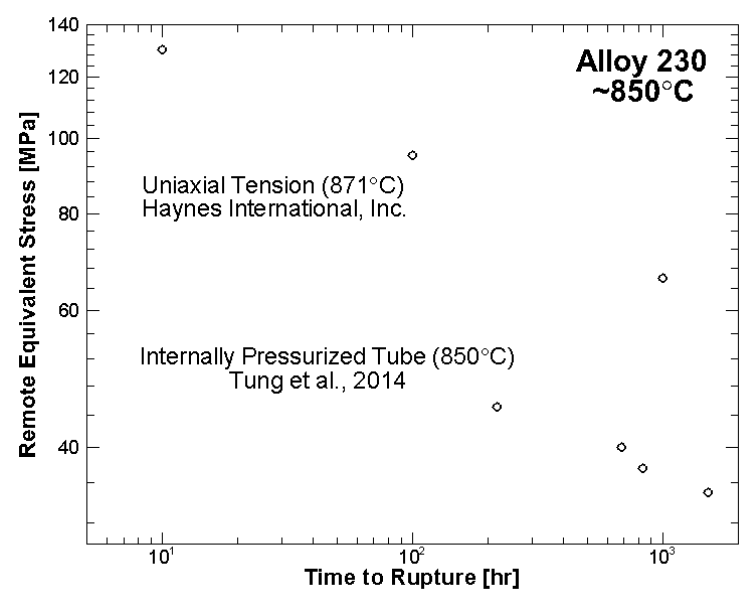

(a)

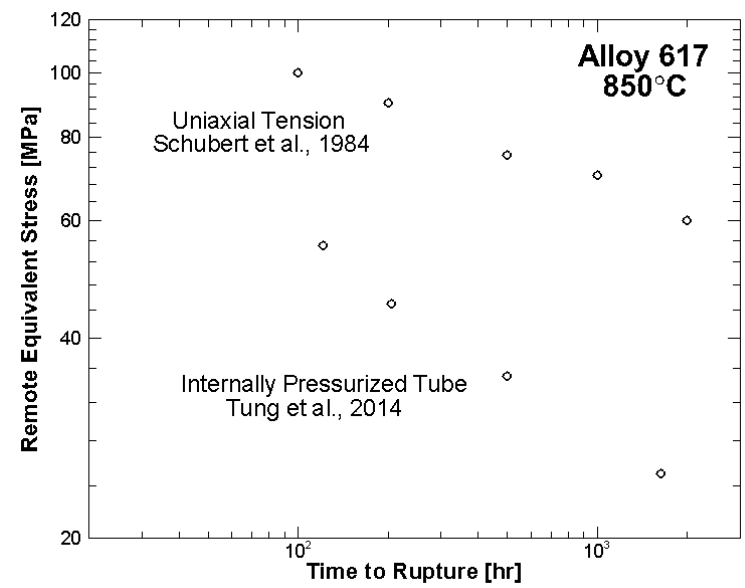

(c)

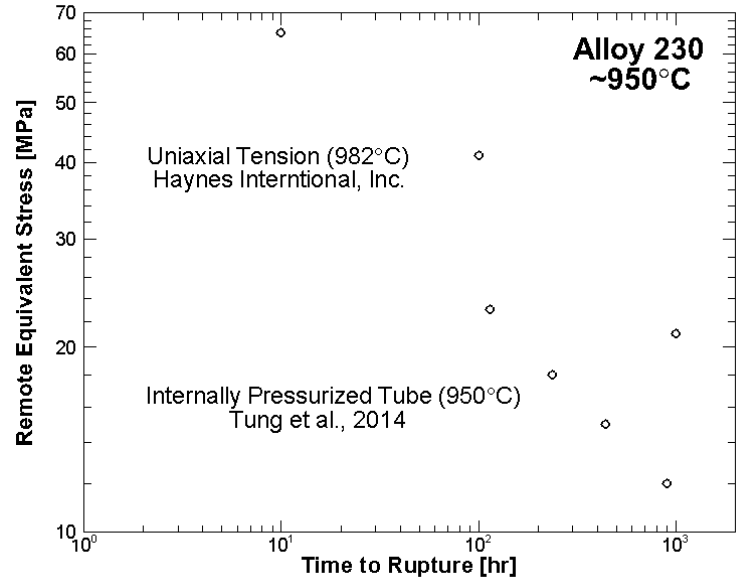

(b)

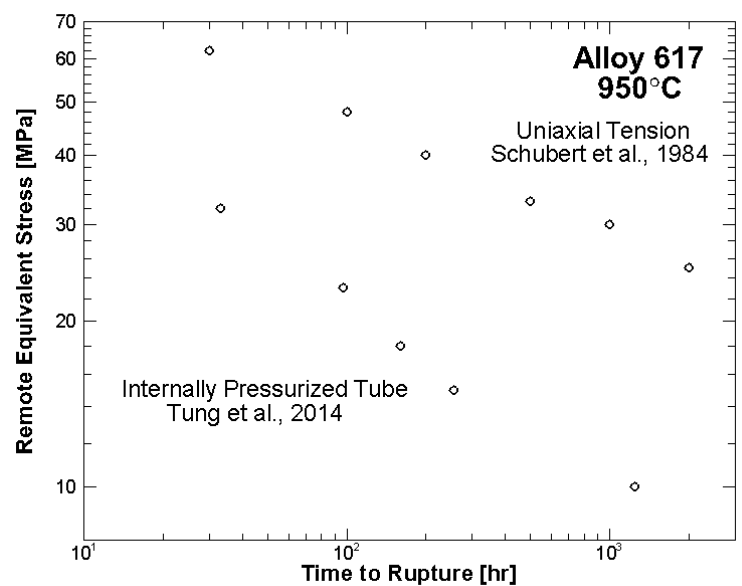

(d)

Figure 1. Experimental remote equivalent stress versus time to rupture data for Alloy 230 at (a) $850^{\circ} \mathrm{C}$ and (b) $950^{\circ} \mathrm{C}$, and Alloy 617 at (c) $850^{\circ} \mathrm{C}$ and (d) $950^{\circ} \mathrm{C}$. Uniaxial data courtesy of Haynes International, Inc. and Schubert et al. [21]. Biaxial data courtesy of Tung et al. [1]. 


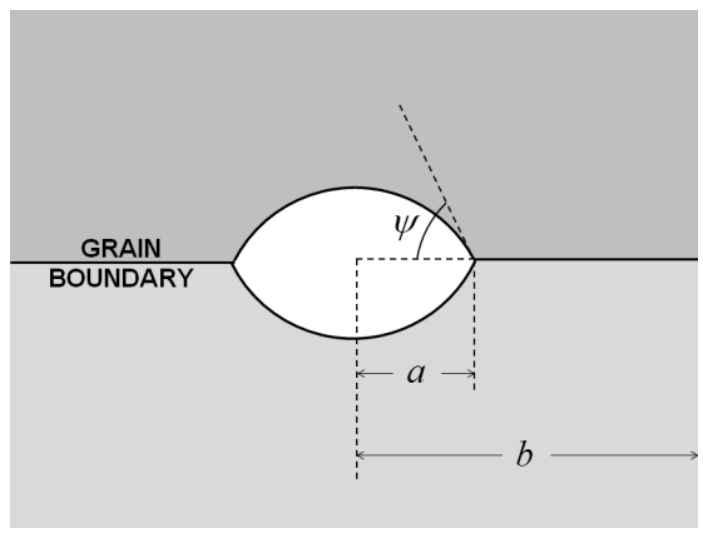

Figure 2. Axisymmetric, spherical cap-shaped void model schematic, illustrating the void radius $a$, mean half-spacing $b$, and angle $\psi$. Adapted from Chuang et al. [6].

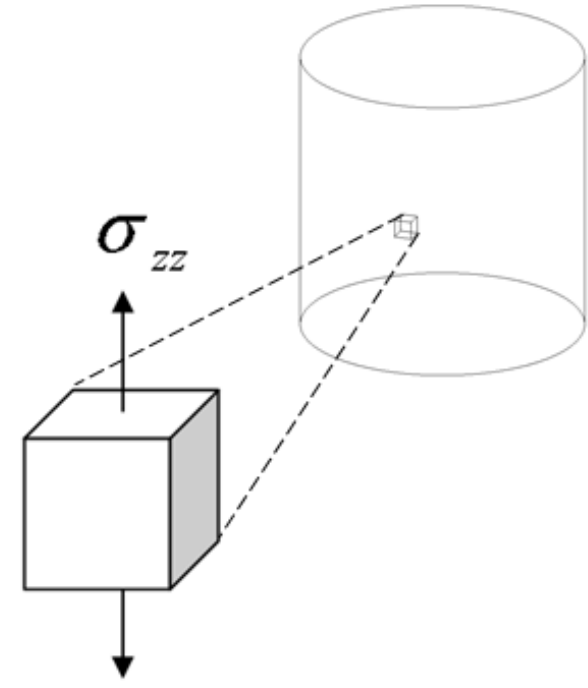

(a)

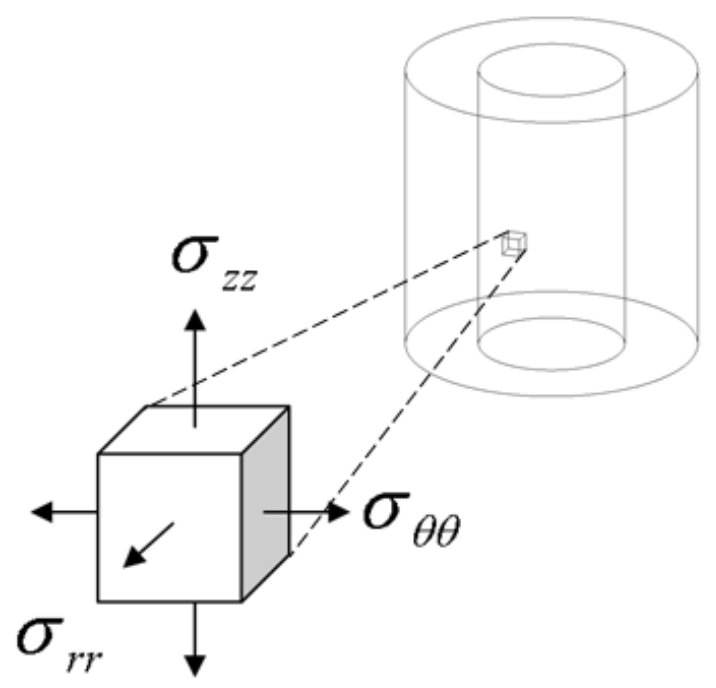

(b)

Figure 3. Infinitesimal material elements illustrating the nonzero stress components for (a) uniaxial tension and (b) biaxial tension within the wall of an internally pressurized, circular cylindrical tube. 


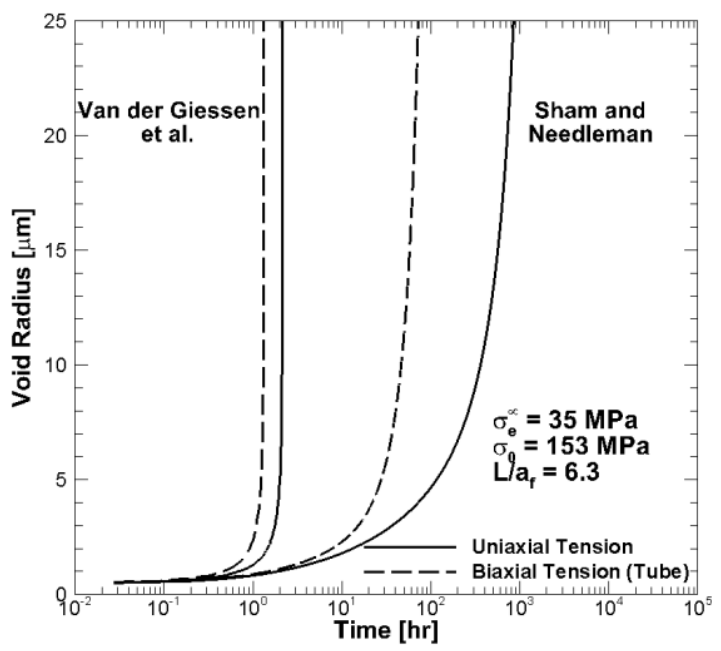

Figure 4. Simulated evolution of void radius over time using the models of Sham and Needleman [9] and Van der Giessen et al. [11] under nominal remote equivalent stress $\sigma_{e}^{\infty}=35$ $\mathrm{MPa}$, creep parameters $\sigma_{0}=153 \mathrm{MPa}, n=6.75$, and $T=800^{\circ} \mathrm{C}$. The other material parameters can be found in Table 1.

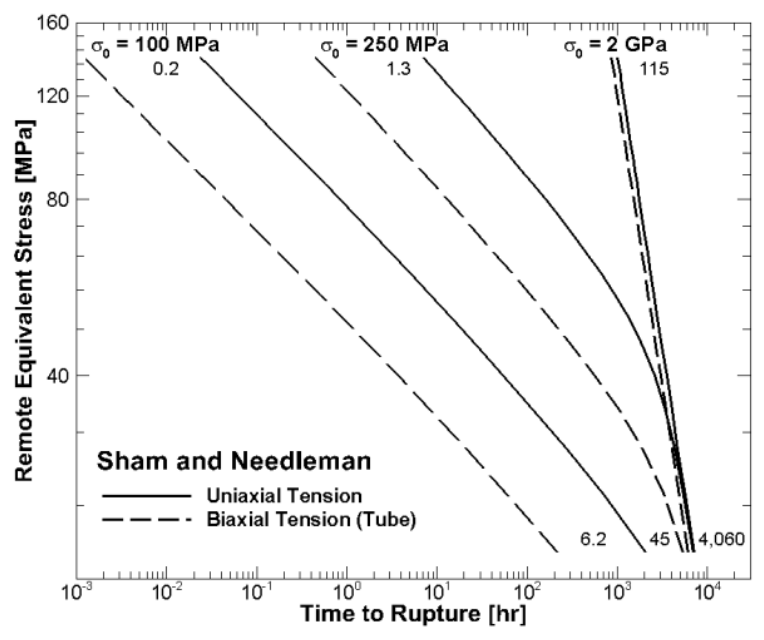

(a)

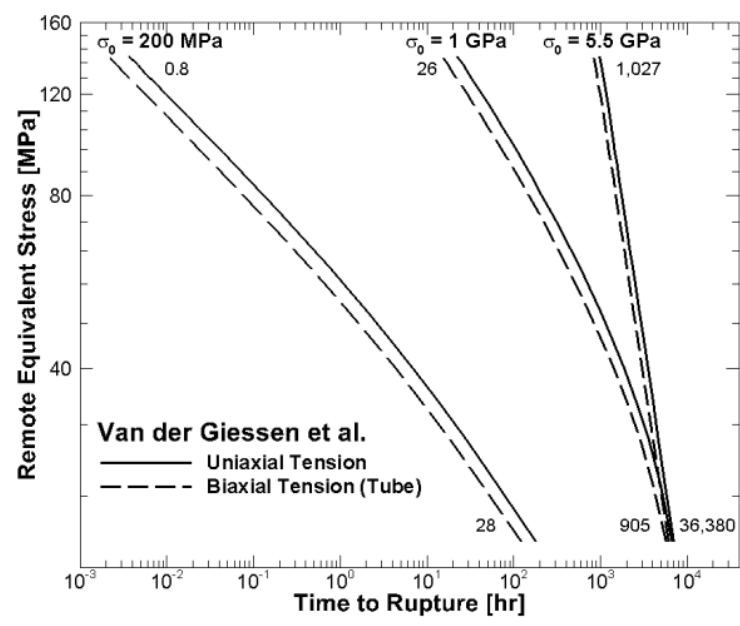

(b)

Figure 5. Simulated nominal remote equivalent stress versus time to rupture for various values of the parameter $\sigma_{0}$ using the models of (a) Sham and Needleman [9] and (b) Van der Giessen et al. [11] with $n=6.5$ and $T=800^{\circ} \mathrm{C}$. The other material parameters can be found in Table 1 . The numbers next to the curves represent the value of the normalized diffusion length $L / a$ at rupture, illustrating bulk creep dominance (the curves with $\sigma_{0}=100 \mathrm{MPa}$ and $\sigma_{0}=200 \mathrm{MPa}$ ), grain boundary diffusion dominance (the curves with $\sigma_{0}=2 \mathrm{GPa}$ and $\sigma_{0}=5.5 \mathrm{GPa}$ ), and comparable bulk creep and grain boundary diffusion (the curves with $\sigma_{0}=250 \mathrm{MPa}$ and $\sigma_{0}=1$ GPa). Nominal stresses are calculated according to (A.19). 


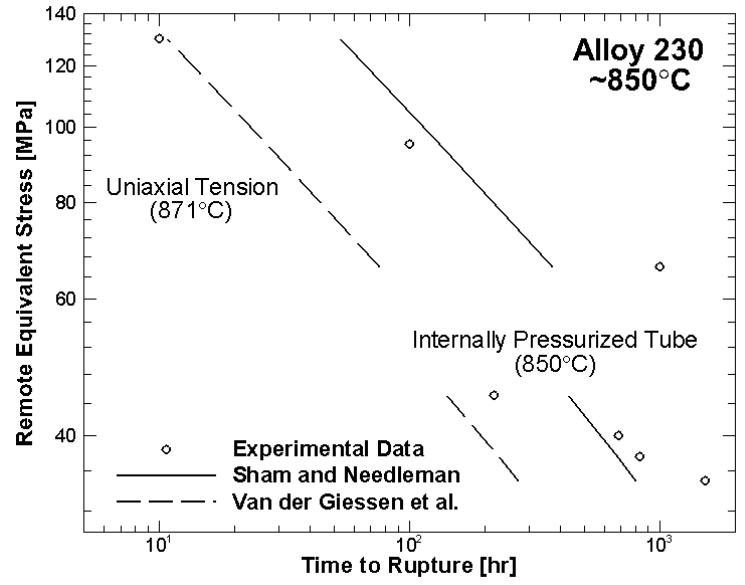

(a)

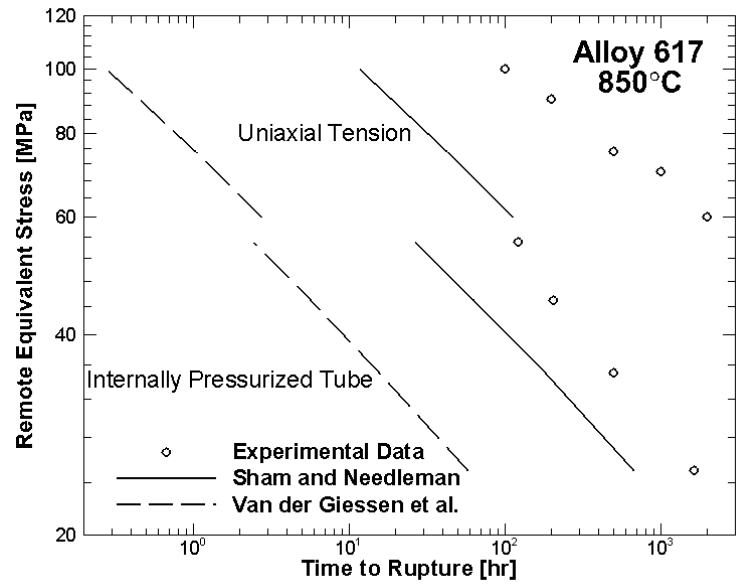

(c)

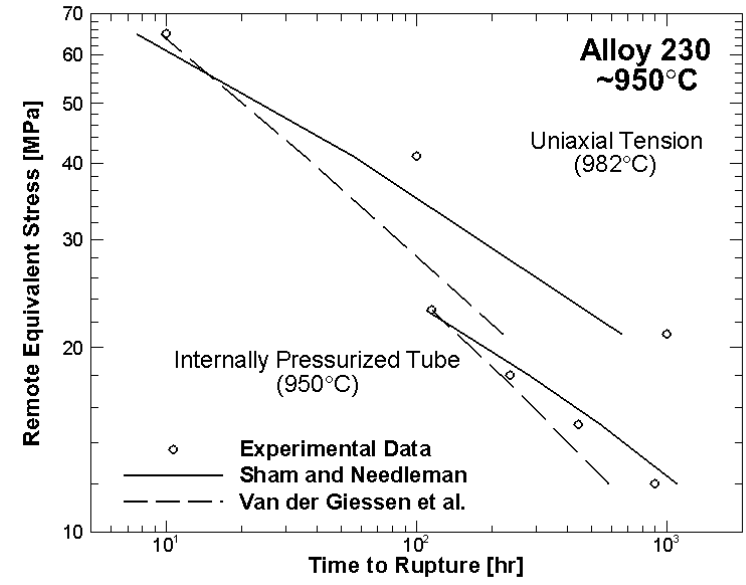

(b)

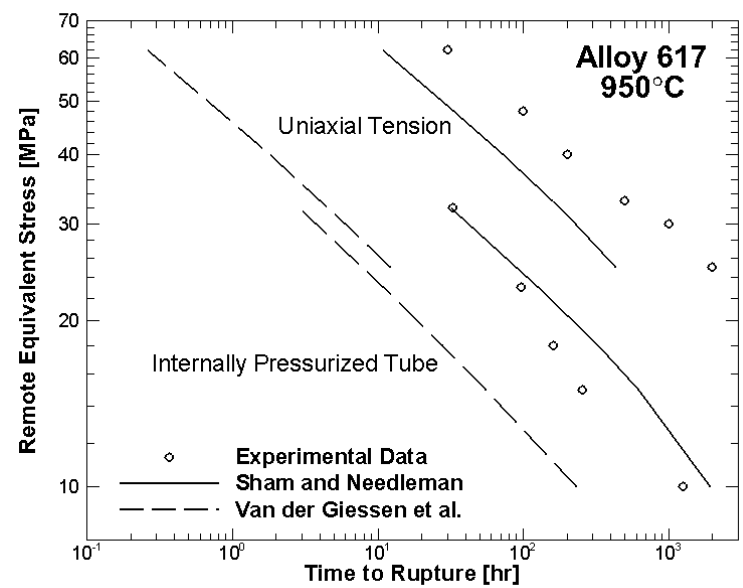

(d)

Figure 6. Simulated nominal remote equivalent stress versus time to rupture for Alloy 230 at (a) $850^{\circ} \mathrm{C}$ and (b) $950^{\circ} \mathrm{C}$, and Alloy 617 at (c) $850^{\circ} \mathrm{C}$ and (d) $950^{\circ} \mathrm{C}$, using the models of Sham and Needleman [9] and Van der Giessen et al. [11], superposed on the data from Figure 1. In each case, the creep parameters $\sigma_{0}$ and $n$ were chosen by least-squares regression, and can be found in Table 4 and Table 5. The other material parameters can be found in Table 1. Nominal stresses are calculated according to (A.19). 